\title{
PROBING HIGHER DIMENSIONS OF HILBERT SPACE IN EXPERIMENT
}

\author{
A. ZeILINGER \\ Institut für Experimentalphysik, Universität Innsbruck \\ Technikerstraße 25, 6020 Innsbruck, Austria
}

\section{Dedicated to Prof. Iwo Biatynicki-Birula on the occasion of his 60th birthday}

Using the correlated pairs produced in spontaneous parametric down-conversion, one can extract quantum states effectively defined in a Hilbert space of any dimension $N$. Furthermore, using just beam splitters and phase shifters one can build any unitary operator in the laboratory. We briefly discuss how this can be done, what kind of states could easily be produced in the laboratory, and we will discuss one explicit result pertaining to photon bunching in an $N$-dimensional Hilbert space.

PACS numbers: 03.65.Bz, 42.50.Wm

\section{Introduction}

Experiments on the foundation of quantum mechanics so far have usually not made use of the full richness of Hilbert space in the sense that many of these experiments can be understood as testing the predictions of quantum mechanics in a Hilbert space of dimension 2 only. This holds for example for most of the neutron interferometry experiments [1], for the Einstein-Podolsky-Rosen and Bell-type experiments [2] or for experiments testing the spinor symmetry [3]. Recently, we have found that using beam splitters one can realize any unitary operator in any IIilbert space of finite dimension [4]. This observation makes a number of novel fundamental experiments possible, for example one can explicitly test the Kochen-Specker paradox [5]. A direction of research, which offers itself immediately, is to use the two-photon states emitted in parametric down-conversion as the radiation subject to such unitary operators in a Ililbert space of higher dimension. In the present paper, we will just give a brief overview of how we propose to produce states in an $N$-dimensional Hilbert space and we will give one explicit example of photon bunching if such states are subject to the general unitary operators.

In the process of down-conversion, a pump beam, typically of UV-energy, interacts with some nonlinear crystal such that with a certain very small probability a UV-photon can split into two photons conserving energy and momentum

$$
k_{1}+k_{2}=k_{0}, \quad \omega_{1}+\omega_{2}=\omega_{0},
$$


where $k_{0}\left(k_{1}, k_{2}\right)$ and $\omega_{0}\left(\omega_{1}, \omega_{2}\right)$ are the wave vector of the pump (down-converted photons) inside the crystal and the frequency of the pump (down-converted photons) respectively. This implies that the frequencies and momenta of the individual photons produced are undefined, they only have to attain values such as to add up to obey Eqs. (1). The conditions of Eqs. (1) are commonly called phase-matching conditions and one should note that they only hold for a sufficiently thick crystal, a condition which we assume here. It then follows from Eqs. (1) that photons of a given wavelength are emitted into a cone whose axis is the pump beam. Furthermore, to each cone with the frequency $\omega_{1}$ there is associated another cone with frequency $\omega_{2}$ such that one photon of a pair produced is always found on the one cone and the other photon on the other cone. In addition, the emission directions of two photons belonging to a pair span a plane containing the pump beam (Fig. 1).

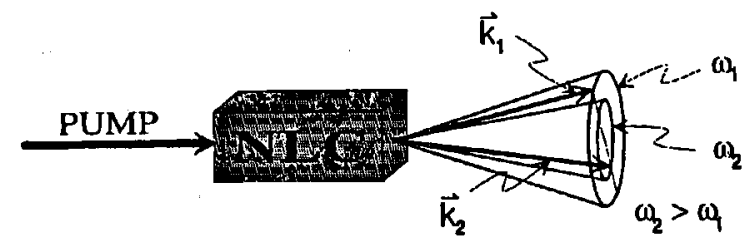

Fig. 1. The principle of parametric down-conversion: a suitable non-linear crystal $(N L C)$ is pumped by a strong laser beam. It then emits photon pairs such that one frequency is emitted into a cone around the pump. One given cone is always associated to another cone, such that energy conservation holds. If one of the two photons is found with, say, momentum $k_{1}$, the other photon is found with momentum $k_{2}$.

It is clear that the state emitted by the nonlinear crystal is one of the richest quantum mechanical entities accessible to direct coherent manipulation in the laboratory. This is because this state (for a representation see [6]) exhibits entanglement both in energy and momentum and because both variables are continuous. All existing experiments make use of only a small part of this rich state by selecting either two or four beams or snatial modes out of the full radiation field generated.

In the next section we will discuss some of the new possibilities of selecting an entangled two-photon state defined in a Hilbert space of higher dimension out of the down-conversion field and of subjecting such a state to unitary transformations.

\section{Multimode entanglement in the laboratory}

There are many ways to extract a correlated two-photon field of a finite number of modes from the radiation produced in down-conversion. Figure 2 gives one such possibility, where for simplicity all modes are extracted from that single cone for which the condition of degenerated down-conversion holds, i.e. $\omega_{1}=\omega_{2}$. $2 N$ pinholes select the output modes $|a\rangle,|b\rangle,|c\rangle \ldots$ The emerging state may thus be written as

$$
|\Psi\rangle=\frac{1}{\sqrt{N}}\{|a\rangle|b\rangle+|c\rangle|d\rangle+|e\rangle|f\rangle+\ldots+|b\rangle|a\rangle+|d\rangle|c\rangle+|f\rangle|e\rangle+\ldots\}
$$




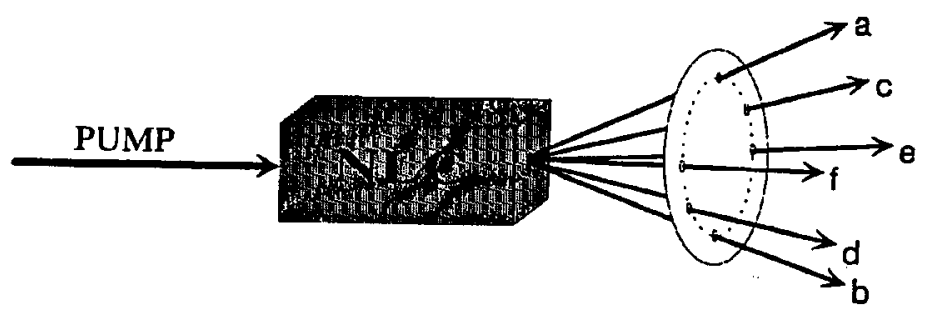

Fig. 2. Using an aperture with suitable pinholes, one can select distinct modes out of the full entangled two-photon state emitted. The figure shows the case of degenerate down-conversion, where both cones collapse into one $\left(\omega_{1}=\omega_{2}\right)$.

where, for example, the ket $|a\rangle$ describes the mode emerging into beam $a$. The second group of terms $(|b\rangle|a\rangle,|d\rangle|c\rangle \ldots)$ are necessary to ensure bosonic symmetry for the whole state. In case we had chosen a source of fermici.., these terms obviously would have had a negative sign. It is evident that $b_{y}$ varying the number of holes, one can vary the number of terms in the entangled state and thus the dimension of the Hilbert space in which the emitted state is defined. We would like to stress once more that the state of Eq. (2), while consisting in principle of any number of modes, contains only two photons. One can then subsequently subject these emerging modes to any apparatus representable by either a unitary or a non-unitary operator and investigate the correlations of the finally emerging radiation.

It has recently been shown [4] that it is possible in the laboratory by just using beam-splitters and phase-shifters to realize experimentally any unitary operator which does not change the number of photons. Without going into the details of this constructive proof, we can therefore state that using standard optical equipment available with today's technology, one can study all phenomena in any finite-dimensional Hilbert space. In practice this can already be realized for one-photon and two-photon states using parametric down-conversion.

An alternative possibility of realizing unitary operators in the laboratory is the use of modern fibre optics technologies. There, it is possible by suitable imaging optics to project just one given mode into a fibre and then to use proper fibre couplers to, again, realize any unitary - or nonunitary for that matter -operator. This technology appears to be less sensitive to external disturbances and more flexible, and it is presently being pursued in our laboratory in Innsbruck. It is particularly suitable for experiments over long distances as for example in future tests of Einstein-Podolsky-Rosen correlations in a truly space-like separated context.

\section{Two particles in $N$ beams}

In this section we will briefly review the possible states of two particles in $N$ beams. For simplicity, again, each beam is considered to be consisting of one mode only and, assuming that all modes have the same spin, we ignore any explicit reference to the spin state. Let these corresponding states be $|a\rangle,|b\rangle,|c\rangle \ldots$ 
Evidently, there are many different ways to distribute two particles over these $N$ states. The first obvious possibility is to have both particles in the same state. This results in the two-particle states

$$
|a\rangle|a\rangle,|b\rangle|b\rangle,|c\rangle|c\rangle, \ldots
$$

There are clearly $N$ such two-photon-one-beam states. Writing a product of kets like $|a\rangle|b\rangle$, we always imply that the first ket refers to particle 1 and the second ket to particle 2. Evidently, the states of Eq. (3) are all bosonic, since they do not change upon permutation of the two particles. Any superposition of these states is also bosonic and thus they span an $N$-dimensional subspace of the total two-particle space of dimension $N^{2}$.

Further base states can be found by considering those cases where the two particles are propagating in two different beams. These states have to be constructed out of a product of different modes. Clearly, a state like $|a\rangle|b\rangle$ is illegal since it obeys neither bosonic nor fermionic symmetry. Possible base states of proper symmetry have therefore to be constructed as superpositions of such product states. Such states are for example

$$
\begin{aligned}
& \frac{1}{\sqrt{2}}\{|a\rangle|b\rangle+|b\rangle|a\rangle\}, \quad \frac{1}{\sqrt{2}}\{|a\rangle|c\rangle+|c\rangle|a\rangle\}, \\
& \frac{1}{\sqrt{2}}\{|b\rangle|c\rangle+|c\rangle|b\rangle\} \quad \text { etc. }
\end{aligned}
$$

These states are symmetric upon interchange of the two particles and therefore bosonic. There are $N(N-1) / 2$ of these bosonic states. Their anti-symmetric counterparts

$$
\begin{aligned}
& \frac{1}{\sqrt{2}}\{|a\rangle|b\rangle-|b\rangle|a\rangle\}, \quad \frac{1}{\sqrt{2}}\{|a\rangle|c\rangle-|c\rangle|a\rangle\}, \\
& \frac{1}{\sqrt{2}}\{|b\rangle|c\rangle-|c\rangle|b\rangle\} \quad \text { etc. }
\end{aligned}
$$

are fermionic. Of these again there are $N(N-1) / 2$ ones.

Altogether, we find that there are $N+N(N-1) / 2=N(N+1) / 2$ bosonic states and $N(N-1) / 2$ fermionic states for two particles in $N$ beams. We would like to stress that in the present discussion we chose a state description rather than a description via operators for the very reason that doing so one can cover both fermions and bosons. Furthermore, we suggest that this provides an approach to the phenomena expected which, even for photons, might lead to an intuitive picture different from that implied by the standard machinery of second quantization quantum optics. For the two-photon experiments considered here the conclusion of the present paragraph is that two photons in $N$ beams are effectively defined in a Hilbert space of dimension $N(N+1) / 2$. Superselection rules prohibit us to reach the $N(N-1) / 2$-dimensional fermionic sector of the two-particle Hilbert space.

\section{An example: two particles in two beams, the Bell states}

From the discussion above it follows that we have the four following possible base states:

$$
\left|\Psi^{+}\right\rangle=\frac{1}{\sqrt{2}}\{|a\rangle|b\rangle+|b\rangle|a\rangle\}, \quad\left|\Psi^{-}\right\rangle=\frac{1}{\sqrt{2}}\{|a\rangle|b\rangle-|b\rangle|a\rangle\}
$$




$$
\left|\Phi_{a}\right\rangle=|a\rangle|a\rangle, \quad\left|\Phi_{b}\right\rangle=|b\rangle|b\rangle .
$$

The states $\left|\Phi_{a}\right\rangle$ and $\left|\Phi_{b}\right\rangle$ can be superposed to form the entangled states

$$
\left|\Phi^{+}\right\rangle=\frac{1}{\sqrt{2}}\{|a\rangle|a\rangle+|b\rangle|b\rangle\}, \quad\left|\Phi^{-}\right\rangle=\frac{1}{\sqrt{2}}\{|a\rangle|a\rangle-|b\rangle|b\rangle\} .
$$

The states $\left|\Psi^{+}\right\rangle,\left|\Psi^{-}\right\rangle,\left|\Phi^{+}\right\rangle$and $\left|\Phi^{-}\right\rangle$together form the so-called Bell basis. They are a complete entangled basis of the two-particle two-dimensional Hilbert space. As we show elsewhere [7], some interesting properties follow when these Bell states are subject to unitary operators acting in these two-dimensional Hilbert spaces. A possible experimental realization of such general unitary operators is simply provided by using variable beam splitters with suitably adjustable phases. Without going into the details of the derivation, we here quote some of the more interesting results.

Firstly, the fermionic state $\left|\Psi^{-}\right\rangle$is an eigenstate of any beam splitter operator. This is a consequence of superselection rules since now the fermionic sector of the Hilbert space is one-dimensional only. Secondly, by choosing the right beam splitter operator, it is possible to turn any of the other Bell states, i.e. the bosonic ones, into each other. Exploiting this property, one can devise a simple system to analyze which state is present. Such systems might also be called "Bell state analyzers", and they might be of relevance, considering experiments on information processing using quantum means. Some particular example would cover quantum teleportation or the fact that one can encode more than one bit into one photon [8-10].

\section{Two photons in a symmetric multiport}

As a symmetric multiport, we define a device which has the following property: if a single particle is incident onto any of its $N$ input ports, it has the same probability, i.e. $P=1 / N$ to emerge at any given output port. This device is the $N$-dimensional analog and generalization [11] of the standard fifty-fifty beam splitter. Of the many novel theoretical results [12] for such a symmetric multiport we just mention one which has already found expcrimental verification. Consider the case where in a multiport with $N$ input ports we have a two-photon state incident into just two input ports $i$ and $j$ :

$$
|\psi\rangle=\frac{1}{\sqrt{2}}\{|i\rangle|j\rangle+|j\rangle|i\rangle\}
$$

where $|i\rangle$ and $|j\rangle$ are modes incident at input $i$ and $j$ respectively (Fig. 3). We now calculate the probability to find both photons together in output $k^{\prime}$. This follows the transformations

$$
\begin{aligned}
& |i\rangle|j\rangle \rightarrow \alpha_{k i} \alpha_{k j}\left|k^{\prime}\right\rangle\left|k^{\prime}\right\rangle, \\
& |j\rangle|i\rangle \rightarrow \alpha_{k j} \alpha_{k i}\left|k^{\prime}\right\rangle\left|k^{\prime}\right\rangle,
\end{aligned}
$$

where e.g. $\alpha_{k i}$ is the transition amplitude from input mode $i$ to output mode $k^{\prime}$. This is the (ij) matrix element of the multiport unitary operator $U$. Thus, the case of finding both photons in output $k$ is described by the state

$$
\left|\psi^{\prime}\right\rangle=\frac{1}{\sqrt{2}}\left(\alpha_{k i} \alpha_{k j}+\alpha_{k j} \alpha_{k i}\right)\left|k^{\prime}\right\rangle\left|k^{\prime}\right\rangle
$$




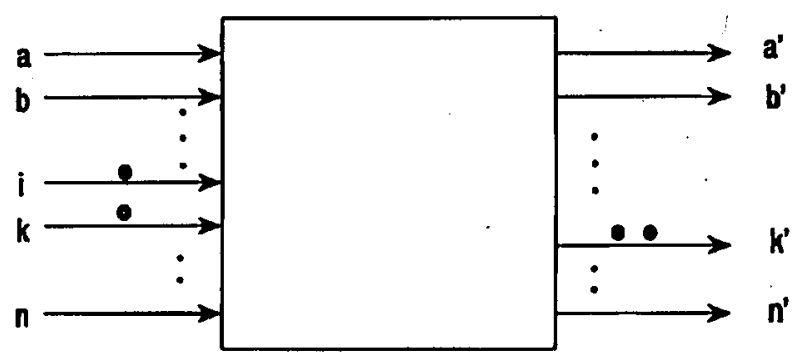

Fig. 3. In a symmetric multiport which can be represented by unitary matrix where all elements have the same modulus, the amplitudes of two photons incident at the two input ports $i, j$ have an increased probability to arrive together at output port $\boldsymbol{k}^{\prime}$.

From the symmetry property of the multiport we obtain $\left|\alpha_{k i}\right|=1 / \sqrt{N}$ and lastly the final state becomes

$$
\left|\psi^{\prime}\right\rangle=\frac{\sqrt{2}}{N} \mathrm{e}^{\mathrm{i} \varphi}\left|k^{\prime}\right\rangle\left|k^{\prime}\right\rangle
$$

where $\varphi$ is an arbitrary phase. Hence we obtain for the probability to find. both photons together in one given output

$$
p \text { (both bosons in a given output })=\frac{2}{N^{2}} .
$$

This probability is enlianced by a factor of 2 over a just statistical arrangement of classical particles, because for classical particles the probability for each particle to arrive at any output is $1 / N$ and therefore the probability of both particles to arrive at the same output is just the square of this probability, i.e.

$$
p \text { (two classical particles in a given output })=\frac{1}{N^{2}} .
$$

Furthermore, it is easy to see that, had the input state been the fermionic state

$$
\left|\psi_{f}\right\rangle=\frac{1}{\sqrt{2}}\{|i\rangle|j\rangle-|j\rangle|i\rangle\}
$$

the probability of both fermions to arrive together in a given output would vanish

$$
p \text { (both fermions in a given output) }=0
$$

as has to be expected from the Pauli exclusion principle.

We would like to stress that we have thus obtained two basic consequences of quantum statistics via a simple interference argument. Finally, we mention that for $N=3$, i.e. the generalization of beam splitters with three input ports and three output ports which we call a tritter [12], the property just mentioned has been demonstrated recently in our laboratory [13]. For $N=2$, i.e. the standard beam splitter, we arrive at the special case that two bosons, for example photons, always arrive together in an output port. This is a well-known property of beam splitters [14] which has already found experimental verification [15]. So far, the spin has entered our considerations only through the symmetry properties of the spatial quantum state. This means that we implicitly assumed that both particles are in the same spin state or, generally speaking, the spin state has even symmetry. In 
a forthcoming paper we will deal with the general case of any symmetry for both the spin state and the spatial state.

\section{Acknowledgments}

This work was supported by the Austrian Science Foundation, grant number S6502 and by the US National Science Foundation, grant \# PHY92-13964.

\section{References}

[1] H. Rauch, W. Treimer, U. Bonse, Phys. Lett. A 47, 369 (1974).

[2] D.M. Greenberger, M.A. Horne, H. Shimony, A. Zeilinger, Am. J. Phys. 58, 1131 (1990).

[3] D.M. Greenberger, Rev. Mlod. Phys. 55, 875 (1983).

[4] M. Reck, A. Zeilinger, H.J. Bernstein, Ph. Bertani, to be published.

[5] S. Kochen, E. Specker, J. Math. Mech. 17, 59 (1967); P. Heywood, M.I.G. Redhead, Found. Phys. 13, 481 (1983).

[6] M. Horne, A. Shimony, A. Zeilinger, in: Quantum Coherence, Ed. J. Anandan, World Scientific, Singapore 1990, p. 356.

[7] A. Zeilinger, J. Mod. Opt., in press.

[8] C.H. Bennett, G. Brassard, C. Crépeau, R. Josza, A. Peres, W.K. Wootters, Phys. Rev. Lelt.70, 1895 (1993).

[9] C.H. Bennett, S.J. Wiesner, Phys. Rev. Lett. 69, 2881 (1992).

[10] H. Weinfurter, Europhys. Lelt., in press.

[11] A. Zeilinger, H.J. Bernstein, D.M. Greenberger, M.A. Horne, M. Zukowski, in: Quantum Control and Measurement, Eds. H. Ezawa, Y. Murayama, Elsevier Science, Amsterdam 1993, p. 9.

[12] A. Zeilinger, M. Zukowski, M.A. Horne, H.J. Bernstein, D.M. Greenberger, paper presented at Fundamental Aspects of Quantum Theory, Columbia, South Carolina, 1992, proceedings in press, Eds. J. Anandan, J.L. Safko, World Scientific 1993.

[13] K. Mattle, diploma thesis, University of Innsbruck, unpublished; K. Mattle, HI. Weinfurter, A. Zeilinger, to be published.

[14] H. Fearn, R. Loudon, J. Opt. Soc. Am. B 6, 917 (1989).

[15] C.K. Hong, Z.Y. Ou, L. Mandel, Phys. Rev. Lett. 59, 2044 (1987). 